\title{
Study on grid adaptability testing methodology for wind turbines
}

\author{
Shaolin LI (ه), Shiyao QIN, Ruiming WANG, \\ Qing LI, Chen CHEN
}

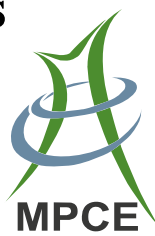

\begin{abstract}
The use of wind power has grown rapidly in recent years. Wind power is a clean source of energy, but can have negative impacts on the distribution grid. The influence of large-scale wind power integration on the safe and stable operation of a power system cannot be ignored. It is necessary and urgent to achieve grid adaptability for wind turbines in China. Using a $35 \mathrm{kV} / 6$ MVA grid simulator, the performance of a grid is investigated by simulation. Typical grid disturbances such as voltage deviation, frequency fluctuation, voltage unbalance, and distortion can be simulated. A grid adaptability testing methodology was developed and applied to a doubly fed wind turbine with a focus on analyzing real test data to ascertain its three-phase voltage unbalance adaptability, which was successfully demonstrated. The methodology can also be used to guide other grid adaptability tests.
\end{abstract}

Keywords Wind turbines, Grid simulator, Grid adaptability testing

\section{Introduction}

In recent years, wind power has undergone rapid development in China. The installed capacity of wind power reached $47 \mathrm{GW}$ by the end of 2011 and is expected to reach $150 \mathrm{GW}$ in 2020 . With the rapid development of wind power, the penetration of its use is increasing [1]. While wind power provides clean energy, it also brings negative impacts to its use on the grid. The influence of

Received: 2 August 2012/ Accepted: 2 November 2012/Published online: 12 July 2013

(C) The Author(s) 2013. This article is published with open access at Springerlink.com

S. LI, S. QIN, R. WANG, Q. LI, C. CHEN, China Electric Power

Research Institute, Beijing 100192, China

$(\bowtie)$ e-mail: lishaolin@epri.sgcc.com.cn large-scale wind power integration on the safe and stable operation of a power system cannot be ignored.

The power quality problems of grid-connected wind turbines are attracting much attention from experts and researchers. In particular, testing theories and methodologies have been developing rapidly, and a large number of papers have been published [2-10]. For example, [2] analyzed the basic principles and influencing factors of grid-connected wind turbine power quality problems, and conclusions were validated by simulation. Reference [3] carried out power quality testing for a wind farm and undertook comparative studies on the characteristics of power quality for different types of wind turbines installed in wind farms. To improve the power quality of grid-connected wind turbines, many different devices and control algorithms have been studied elsewhere [4]. In addition, IEC 61400-21: 2008 and GB/T 20320-2006 have standardized the power quality testing and evaluation methods. At the same time, wind turbine manufacturers and testing institutes have carried out many power quality tests on wind turbines.

It is clear that some interactions exist between wind turbines and a power system. As a part of the power system, wind turbines are not only one of the causes of the power quality problems but also they themselves suffer from grid disturbances. Therefore, wind turbines need to be able to adapt to grid disturbances.

There are clear requirements for grid adaptability of wind farms in the associated National Standard of China [11] which specifies the operating range of the voltage and frequency of a wind farm, and specifies the tolerance levels for voltage unbalance, flicker. and harmonics. However, in China, there is a lack of research on the theory of grid adaptability and the method and devices for testing it. The progress on grid adaptability testing for wind turbines or wind farms is very slow. At present, most wind turbines run 
in the power system without grid adaptability testing, and so the safe and stable operation of the power system is under threat. For example, at the beginning of 2011, a grid fault occurred in the northwest of China. This accident initially caused fluctuation of the grid voltage, dropping the grid frequency, which led to numbers of wind turbines disconnecting from the grid. The accident caused a serious threat to the safe and stable operation of the power system. Accident reporting and investigation showed that poor grid adaptability of wind turbines was the main reason for this accident, which emphasized the necessity and urgency for grid adaptability testing of wind turbines.

In this study, a $35 \mathrm{kV} / 6$ MVA grid simulator was first introduced to enable grid adaptability testing of wind turbines. A grid simulator can generate voltage deviations, frequency fluctuations, three-phase voltage unbalance, flicker, voltage distortions, and other common grid disturbances. The output characteristics of the grid simulator were then analyzed by simulation, which could confirm whether the turbine met the grid adaptability testing requirements. Finally, the principles and methodology of wind turbine grid adaptability testing were discussed. A case study of a grid adaptability test for a doubly fed wind turbine is described with a focus on analyzing the real test data of three-phase voltage unbalance adaptability. The methodology can also be used to guide other grid adaptability tests.

\section{Grid simulator}

\subsection{Design of grid simulator system}

The grid simulator that is used to test the grid adaptability of a wind turbine needs to include the following basic functions:

(1) It must simulate and generate the basic grid disturbances, such as voltage deviation, frequency fluctuation, voltage unbalance, flicker, voltage distortion, and other common grid disturbances. Furthermore, the disturbances must meet typical testing requirements.

(2) It must have the ability to operate in four-quadrants, and the power must be able to flow in both directions, so that the tested wind turbine can freely transfer from a power-consumption state to a power-generation state.

(3) The grid simulator should not affect the access point on the grid in either power-consuming or -generating states.

Based on these requirements, we developed a set of highvoltage and large-capacity devices to test the grid adaptability of a wind turbine, i.e., $35 \mathrm{kV} / 6$ MVA grid simulator. The schematic diagram of the grid simulator is shown in
Fig. 1. The device is mainly composed of two parts: consisting of low- and high-frequency disturbance-generating devices. The low-frequency disturbance-generating device can generate the voltage deviation, frequency fluctuation, voltage unbalance, flicker and other common low-frequency disturbance states. The high-frequency disturbance-generating device can generate the high-order harmonics and voltage distortions.

The low-frequency disturbance-generating device is based on back-to-back converter technology [12]. It includes a step-down transformer, back-to-back converter, and step-up transformer. The schematic diagram of this device is shown in Fig. 2.

The working principles for the low-frequency disturbancegenerating device are summarized as follows. The step-down transformer transfers the medium-voltage to $\mathrm{U} 1$, which is the working voltage of the back-to-back converter, and the converter outputs the desired disturbance voltage waveform according to the modulation wave signal. The step-up transformer then transfers the low voltage level disturbance voltage to the medium-voltage level. Finally, the disturbance voltage is realized at the high side of the wind turbine transformer. The grid adaptability test can then be performed. Because of the back-to-back converter, the wind turbine is completely isolated from network, and so the disturbances cannot affect the voltage at the grid access point. At the same time, the output of disturbance voltage can be changed freely by modifying the modulation wave signal.

Because of the restriction of the converter interfacing filter, the low-frequency disturbance-generating device is not suitable for simulating voltage harmonics and distortions. However, according to the grid code requirements, the wind turbine should be able to tolerate voltage harmonic disturbances. Therefore, according to the principle of serial voltage sources, a high-order harmonic

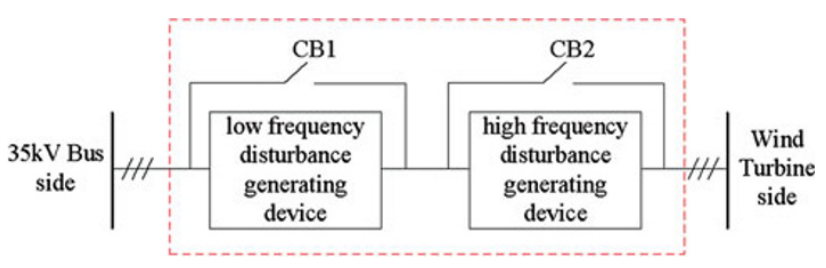

Fig. 1 Schematic diagram of grid simulator

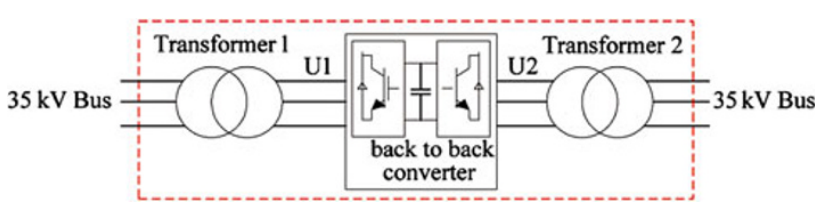

Fig. 2 Schematic diagram of the low-frequency disturbance-generating device 
disturbance was superimposed on the output of the lowfrequency disturbance-generating device, and the harmonics were realized. The high-frequency disturbance-generating device was also based on single-phase back-to-back converter technology, and the inverter side of the $\mathrm{H}$ bridge could be used in a cascade mode [13] to enhance the voltage levels. As mentioned earlier, by modifying the modulation wave signal of the inverter, the corresponding high-voltage harmonics can be generated on the $35 \mathrm{kV}$ bus.

\subsection{Simulation study of grid simulator}

To study and verify the output characteristics of the grid simulator, a grid simulation model based on Fig. 1 was built. The output characteristics of the grid simulator were researched by simulation.

Figure 3 shows the simulation voltage wave when the grid simulator generated a voltage deviation of $-15 \%$, where the dotted line is the output voltage of the grid simulator and the solid line is the voltage at the grid access point. We see that the grid simulator can output the deviation of the voltage in accordance with the set value. Furthermore, it does not affect the voltage at the grid access point. The grid simulator has a voltage adjustment range from $20 \% U_{\mathrm{n}}$ to $130 \% U_{\mathrm{n}}$ with a resolution of $0.5 \% U_{\mathrm{n}}$ and the rate of voltage regulation can be set freely. It can generate the voltage operating range of the wind farm stated by the grid code. Therefore, it can be used for voltage adaptability testing of a wind turbine.

Figure 4 shows the simulation voltage wave when the grid simulator outputs a voltage frequency of which is $62 \mathrm{~Hz}$; the dotted line is the output voltage of the grid simulator, and the solid line is the voltage wave at the grid access point. The grid simulator has a frequency adjustment range from 45 to $66 \mathrm{~Hz}$ with a resolution of $0.01 \mathrm{~Hz}$, and the rate of frequency regulation can be set freely.

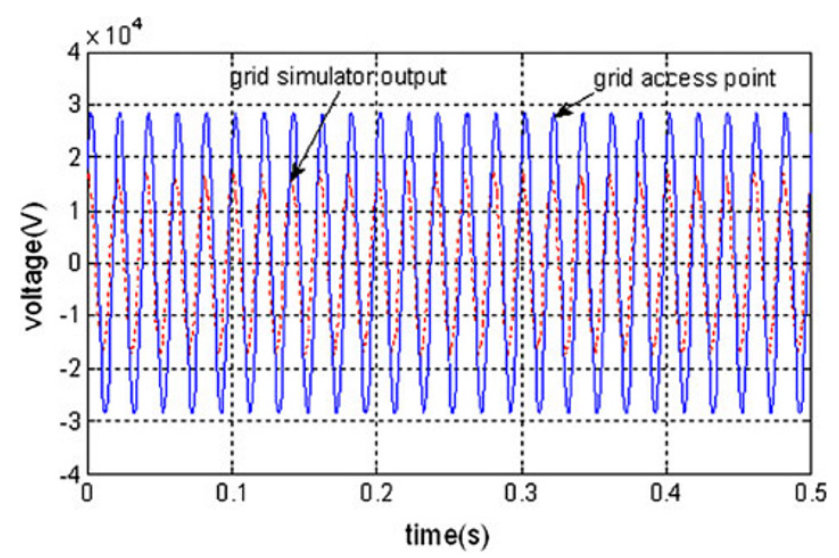

Fig. 3 Simulation wave of voltage deviation

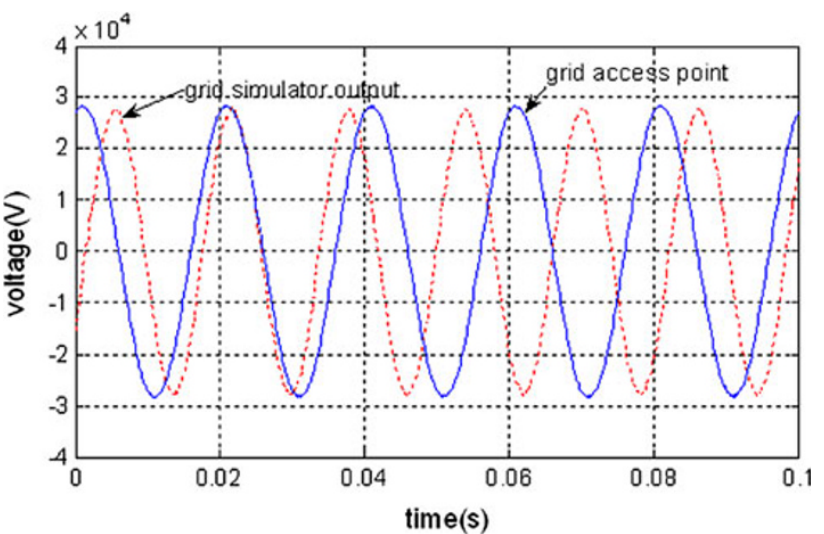

Fig. 4 Simulation wave of frequency deviation

Figure 5 shows the simulation voltage wave when the grid simulator outputs a voltage fluctuation amplitude of which is $\pm 10 \%$ and fluctuation frequency is $10 \mathrm{~Hz}$. The dotted line is the output voltage of the grid simulator, and the solid line is the voltage at the grid access point. The fluctuation amplitude was within $\pm 10 \% U_{\mathrm{n}}$, and frequency fluctuation was from 0 to $25 \mathrm{~Hz}$. Different voltage flicker disturbances can be generated through different combinations of voltage and frequency fluctuations.

Figure 6 shows the simulation wave when the grid simulator outputs a three-phase unbalanced voltage. Figure 6a shows the voltage at the grid access point when the voltage unbalance factor of the grid simulator was set to $7.5 \%$. Figure $6 \mathrm{~b}$ shows the grid simulator output voltage. Figure $6 \mathrm{c}$ shows the output voltage unbalance factor corresponding to Fig. 6a. The grid simulator has a voltage unbalance factor adjustment ranging from $0.5 \%$ to $10 \%$ with a resolution of $0.1 \%$. It can be used to test the voltage unbalance-tolerance ability of a wind turbine.

Figure 7 shows the simulations of voltage wave and harmonic content of the voltage when the grid simulator output the 16th harmonic voltage, and the harmonic ratio

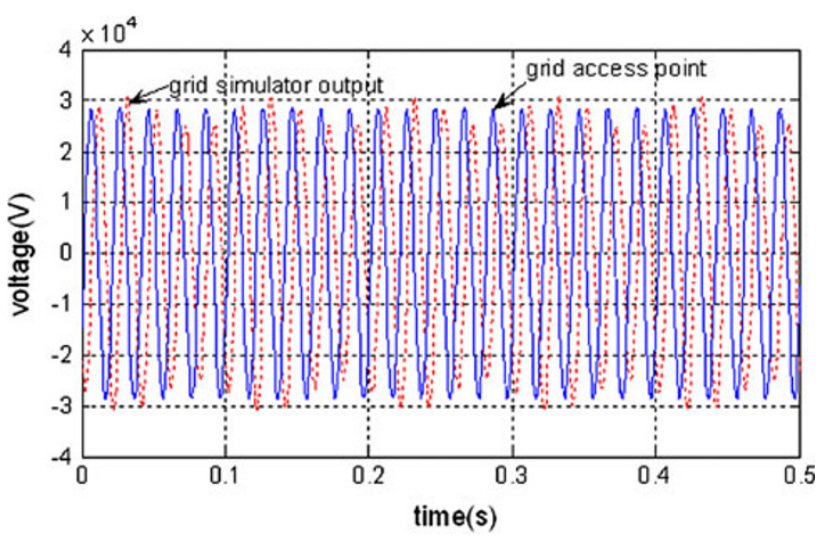

Fig. 5 Simulation wave of voltage fluctuation and flicker 
(a)

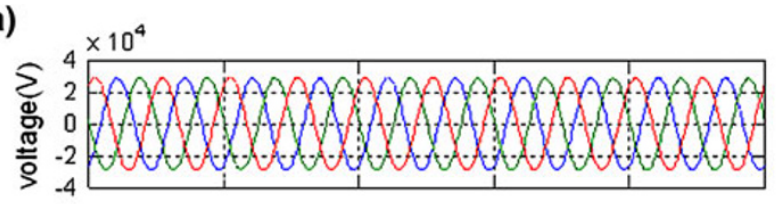

(b)

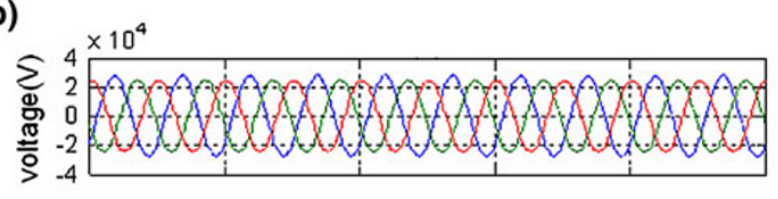

(c)

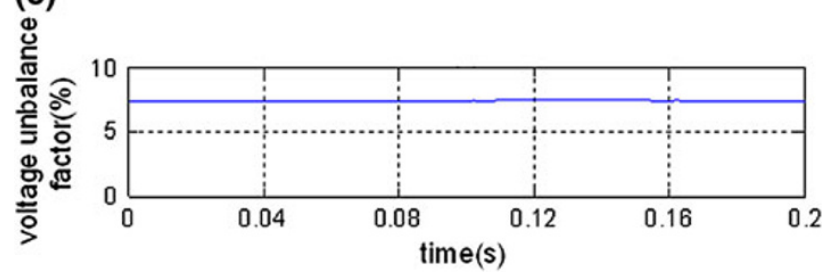

Fig. 6 Simulation wave of three-phase voltage unbalance
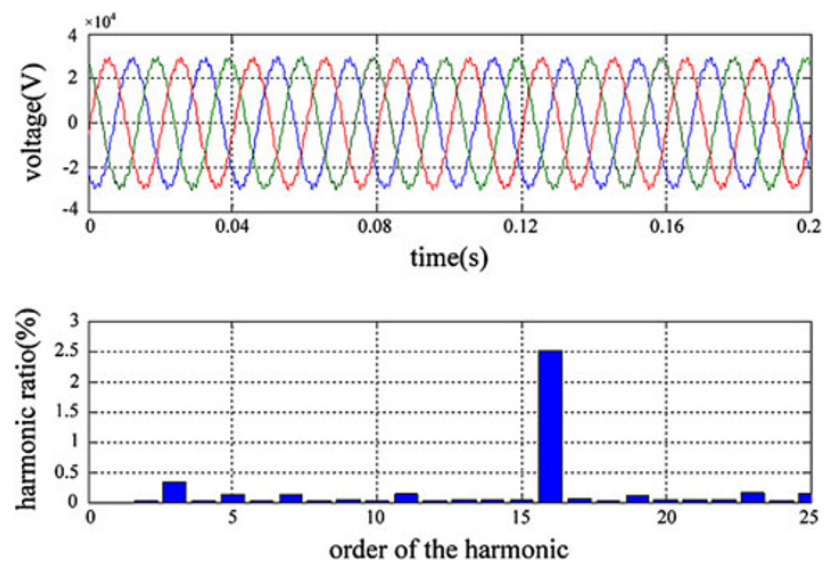

Fig. 7 Simulation wave of voltage harmonics and distortion

for the voltage was set to $2.5 \%$. The grid simulator can generate from 2 nd to 25 th voltage harmonics. A variety of voltage distortions can be imitated through the combinations of the various voltage harmonics. Therefore, it can be used to test the harmonic-tolerance ability and anti-jamming capability of a wind turbine.

\section{Grid adaptability test for a wind turbine}

3.1 The principle of grid adaptability test for a wind turbine

The schematic diagram for a wind turbine grid adaptability test is shown in Fig. 8.

The grid simulator was connected in series between the high-voltage side of the wind turbine transformer and

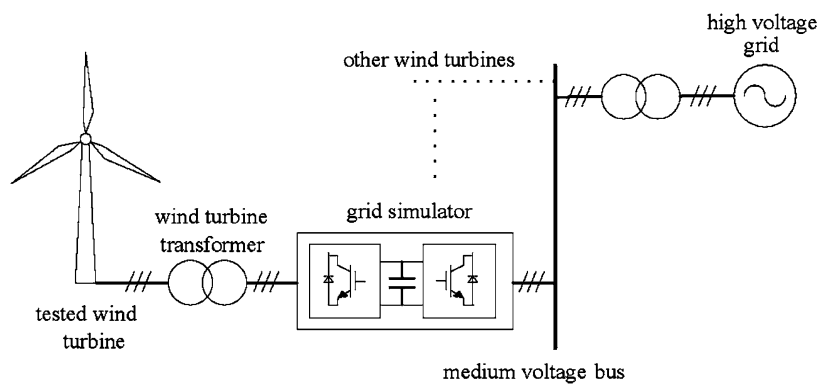

Fig. 8 Test schematic diagram for grid adaptability testing

Table 1 Testing points for the voltage unbalance adaptability test

\begin{tabular}{ll}
\hline Three-phase voltage unbalance factor $(\%)$ & Lasting time $(\mathrm{min})$ \\
\hline 2.0 & $\geq 10$ \\
4.0 & $\geq 1$ \\
\hline
\end{tabular}

medium-voltage bus. The tested wind turbine should be in normal operation during the grid adaptability test. We can then impose various grid disturbances on the high-voltage side of the wind turbine transformer using the grid simulator.

During the process of wind turbine grid adaptability testing, the voltage and current signals at both the highvoltage side of the wind turbine transformer and terminal should be measured. Furthermore, the real-time wind speed signal is very important for verifying the operating status of the tested wind turbine, by analyzing the test data to judge its operating range and protection configuration. The grid adaptability of the tested wind turbine can then be understood.

\subsection{Test of voltage unbalance adaptability for wind turbine}

Wind turbine grid adaptability testing includes the tests for adaptability to voltage deviation, frequency deviation, three-phase voltage unbalance, voltage fluctuation, and flicker and harmonics.

An example of a voltage unbalance adaptability test for a 1.5-MW doubly fed wind turbine is presented to illustrate the test procedure for a grid adaptability test. In addition, it includes the testing contents and testing methods. This will be useful for reference and guidance on the other grid adaptability tests.

We formulate the test procedure according to relevant standards. For voltage unbalance, the National Standard of China "Technical rule for connecting a wind farm to a power system" requires that the voltage unbalance-tolerance ability of a wind farm should meet the National 
Standard of China "GB/T 15543", It prescribes that the electrical and power generation equipment (including the wind turbine) should be in normal operational status when the voltage unbalance factor reaches $2 \%$ and can be run for a short term when the voltage unbalance factor reaches $4 \%$. The testing content and procedure of the voltage unbalance adaptability are clear, and the test points are shown in Table 1.

The voltage unbalance adaptability testing of a wind turbine can be divided into three steps. The first is the noload test, in which it is necessary to confirm that the grid simulator can generate the required voltage disturbance waveforms, because the operation of the wind turbine may cause voltage changes at the grid access point.

The second is the load test without disturbances. The operating characteristics of the wind turbine should be studied when the grid simulator generates the normal grid voltage, so that we can analyze how the asymmetries of transmission line, transformer, and generator parameters affect the operating characteristics of the tested wind turbine, and especially its influence on the three-phase current. Figure 9 shows the experimental curves in this condition. Figure 9a shows the real-time waveform of the fundamental voltage generated by the grid simulator, and Fig. $9 \mathrm{~b}$ shows the corresponding three-phase voltage unbalance factor. The grid simulator has a good balanced voltage output capability, and the voltage unbalance factor is only $0.2 \%$.

It can be seen in Fig. 9c that the wind turbine was running in good condition and the active power was fluctuating according to the wind speed. In Fig. 9d, we can see that the corresponding three-phase current unbalance factor was about $1.0 \%$, and the current unbalance factor was approximately in inverse proportion to the wind speed.

The third step was a load test when the grid simulator produced a three-phase unbalanced voltage. Figure 10 shows the experimental curves of the tested wind turbine (a)

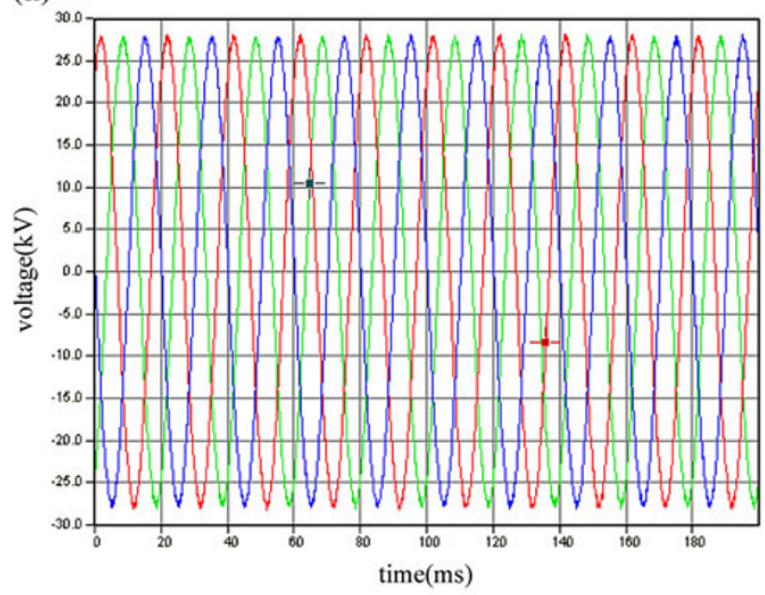

(c)

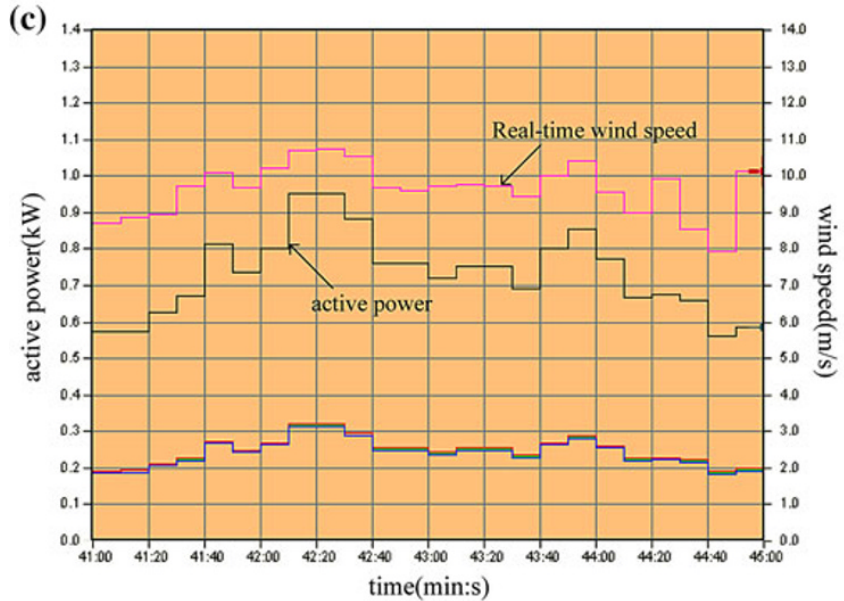

(b)

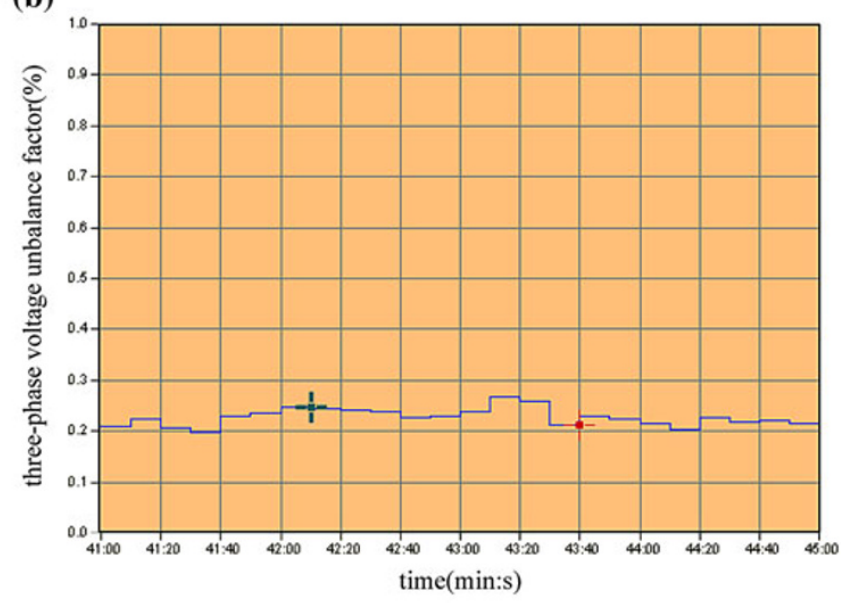

(d)

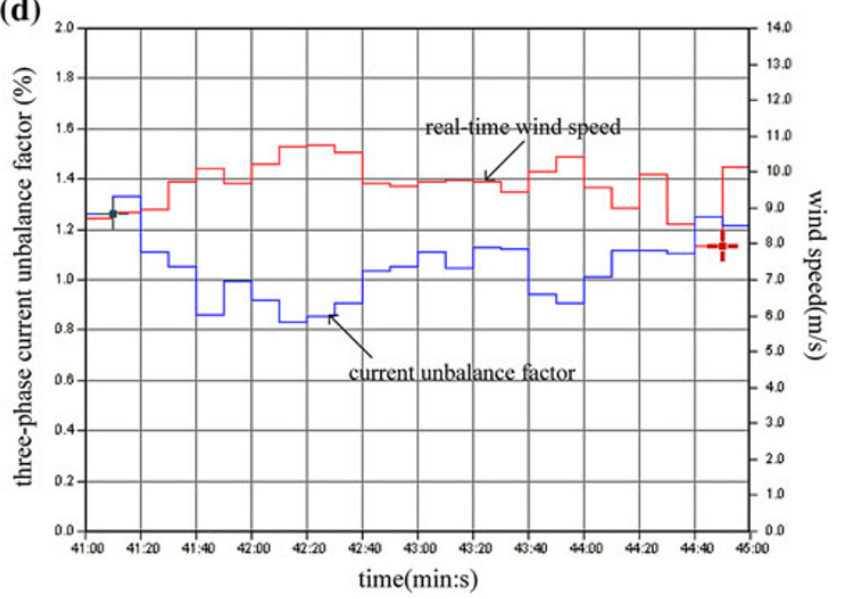

Fig. 9 Operating characteristics of the wind turbine without disturbance. a Voltage wave on the high-voltage side of the wind turbine transformer. b Voltage unbalance factor on the high-voltage side of the wind turbine transformer. $\mathbf{c}$ Relationship between the output active power and wind speed. d Relationship between the current unbalance factor and wind speed 
(a)

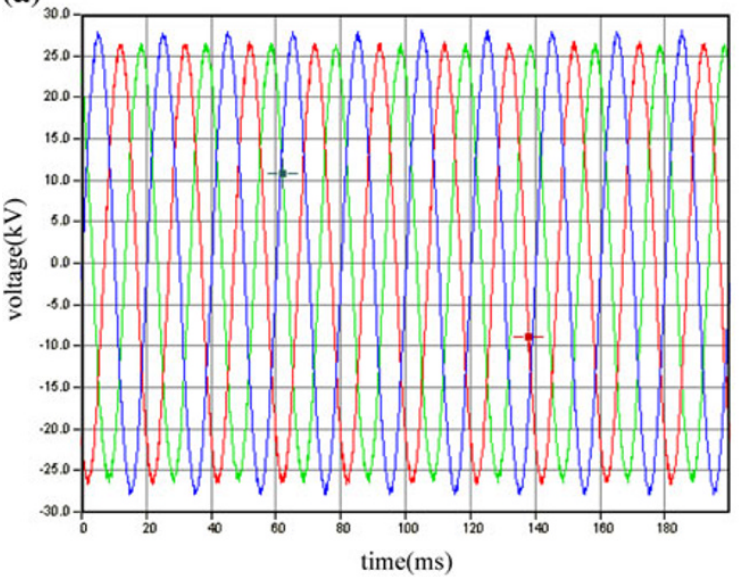

(c)

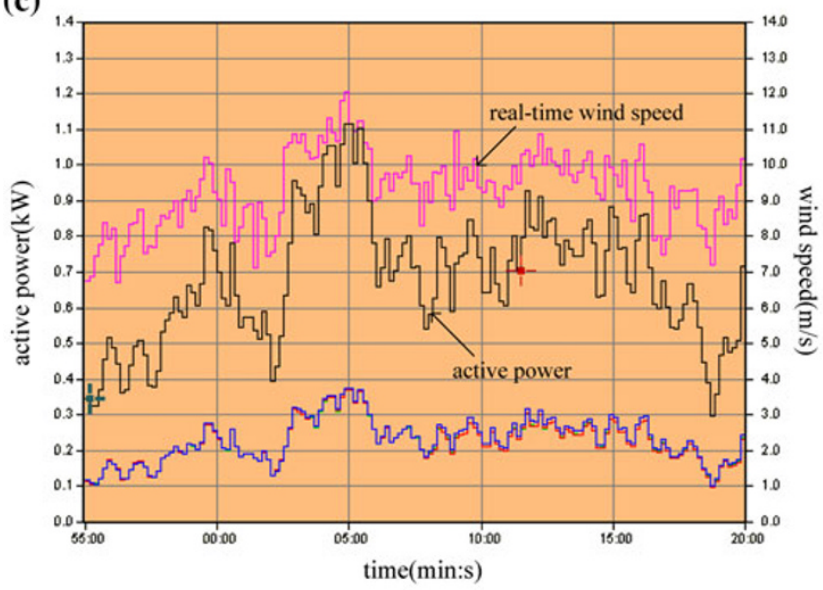

(b)

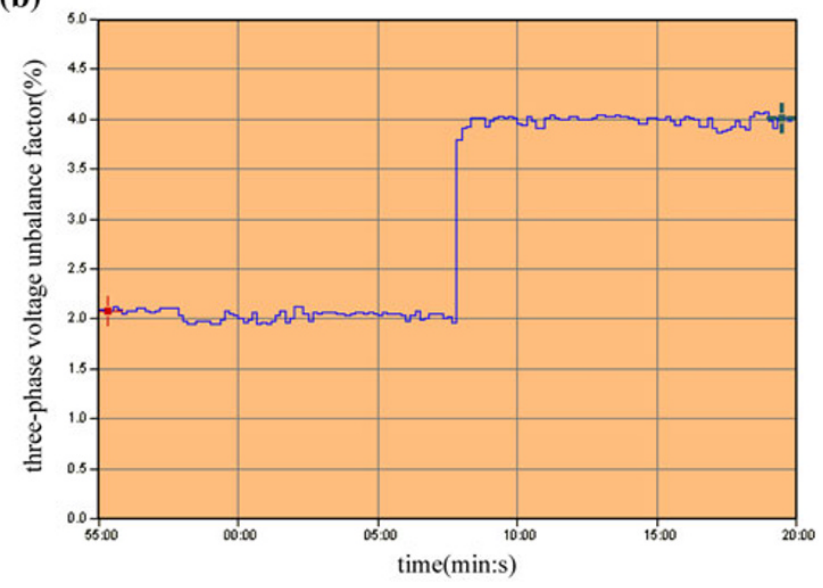

(d)

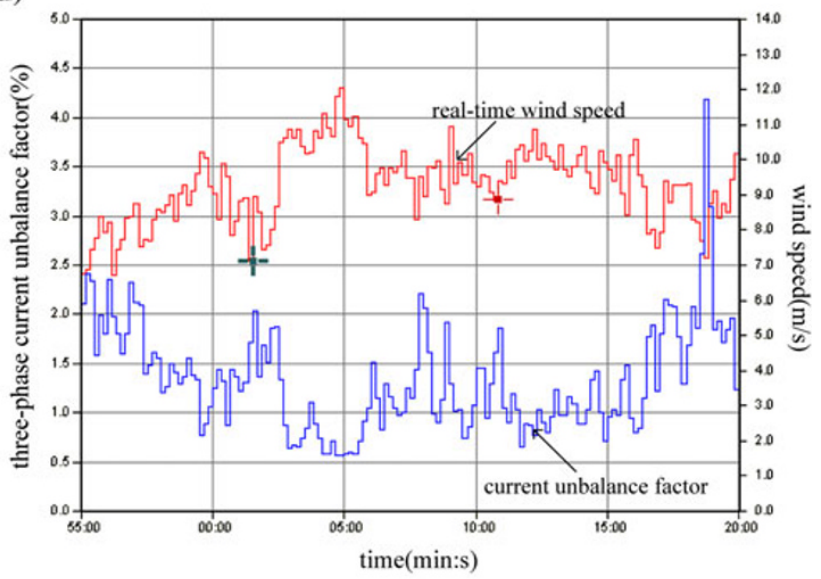

Fig. 10 Operating characteristics of the wind turbine under three-phase voltage unbalance disturbance imposed. a Voltage wave on the high-voltage side of the wind turbine transformer. $\mathbf{b}$ Voltage unbalance factor on the high-voltage side of the wind turbine transformer. $\mathbf{c}$ Relationship between the output active power and wind speed. $\mathbf{d}$ Relationship between the current unbalance factor and wind speed

when the voltage unbalance adaptability testing was carried out.

The three-phase voltage unbalance factor of the grid simulator was $2.0 \%$ at the beginning, and 15 min later, it changed to $4.0 \%$. Figure 10a shows the real-time voltage waveform of the grid simulator output parameter of which for the three-phase voltage unbalance factor was set to $4.0 \%$. The online calculated three-phase voltage unbalance factor is shown in Fig. 10b. It can be seen in Fig. 10c that the wind turbine was in good running condition and the active power fluctuated according to the wind speed in the whole process of changing the voltage unbalance condition. In addition, in Fig. 10d we can see that the threephase current unbalance factor in this process was about $1.3 \%$ which changed little compared with Fig. 9d.

The test results show that the tested doubly fed wind turbine had an excellent ability to control the voltage unbalance, and avoided the negative impact of the unbalance voltage on the induction generator and the rotor converter such as high levels of unbalanced currents, pulsating torques, overheated windings, and so on. Therefore, we concluded that the tested wind turbine had a good tolerance to three-phase voltage unbalance.

In summary, the voltage unbalance adaptability of the tested wind turbine was in good order, and it could withstand a three-phase voltage unbalance factor up to $4.0 \%$, thus meeting the China grid code. This indicates that the methodology could also be used as guidance for other grid adaptability tests.

\section{Conclusions}

The necessity and the urgency for grid adaptability testing of wind turbines in China are first discussed. A $35 \mathrm{kV} /$ 6 MVA grid simulator was then introduced, and the 
performance of grid simulator was studied by simulation. Finally, the principles and methodology for grid adaptability testing were discussed. A case study of a grid adaptability test for a doubly fed wind turbine was carried out. The methodology could also be used to guide other grid adaptability tests. Further study involves developing a mobile $35 \mathrm{kV} / 6 \mathrm{MVA}$ grid simulator to facilitate grid adaptability testing of wind turbines which are situated in different wind farms and thoroughly studying the principles and methodology of grid adaptability testing of wind turbines.

Open Access This article is distributed under the terms of the Creative Commons Attribution License which permits any use, distribution, and reproduction in any medium, provided the original author(s) and the source are credited.

\section{References}

[1] Teninge A, Roye D, Bacha S (2010) Reactive power control for variable speed wind turbines to low voltage ride through grid code compliance. In: Proceedings of the 19th international conference on electrical machines (ICEM'10), Rome, Italy, 6-8 Sept 2010

[2] Linh NT (2009) Power quality investigation of grid connected wind turbines. In: Proceedings of the 4th IEEE conference on industrial electronic and applications (ICIEA'09), Xi'an, China, 25-27 May 2009, pp 2218-2222

[3] Chen Bing, Yuan Xiaodong, Xu Yang et al. (2010) Power quality measurement and comparison between two wind farms equipped with FSIG + PMSG and DFIG. In: Proceedings of the 2010 international conference on power system technology: Technological innovations making power grid smarter (POWERCON'10), Hangzhou, China, 24-28 Oct 2010

[4] Mohod SW, Aware MV (2008) Power quality issues \& it's mitigation technique in wind energy conversion. In: Proceedings of the 13th international conference on quality power \& harmonic (ICHQP'08), Wollongong, Australia, 28 Sept-1 Oct 2008

[5] Gallo D, Landive C, Pasquino N (2008) An instrument for objective measurement of light flicker. Measurement 41(3):334-340

[6] Georgilakis PS (2008) Technical challenges associated with the integration of wind power into power systems. Renew Sustain Energy Rev 12(3):852-863

[7] Mutlu OS, Akpinar E, Baliker A (2009) Power quality analysis of wind farm connected to Alacati substation in Turkey. Renew Energy 34(5):1312-1318

[8] Sorensen P, Cutululis NA, Lund T et al. (2007) Power quality issues on wind power installations in Denmark. In: Proceedings of the 2007 IEEE Power Engineering Society general meeting, Tampa, FL, 24-28 June 2007

[9] Brekken TKA, Mohan N (2007) Control of a doubly fed induction wind generator under unbalanced grid voltage conditions. IEEE Trans Energy Convers 22(1):129-135

[10] IEC 61400-21:2008 Wind turbines-Part 21: Measurement and assessment of power quality characteristics of grid connected wind turbines, 2008

[11] GB/T 19963-2011 Technical rule for connecting wind farm to power system, 2011 (in Chinese)

[12] Zhaoan Wang, Jun Huang (2000) Power electronics technology, 4th edn. Mechanical Industry Press, Beijing (in Chinese)

[13] Rodriguez J, Lai J, Fang ZP (2002) Multilevel inverters: a survey of topologies, controls, and applications. IEEE Trans Ind Electron 49(4):724-738

\section{Author Biographies}

Shaolin LI received his master degree in Control Theory and Control Engineering from Hefei University of Technology in 2010. He is presently working on renewable energy power generation technology. His research interests include wind power grid integration test technology and power electronics. E-mail:1s11984a@163.com.

Shiyao QIN is a senior electrical engineer, deputy director of renewable energy department of CEPRI. He is a member of the National Wind Mechanical Standardization Committee. His research interests include wind power grid integration test technology and motor control.

Ruiming WANG received his master degree in Power System from North China Electric Power University (Beijing) in 2004. He is now working in China Electric Power Research Institute (CEPRI). His research interests include wind power grid integration test technology and power system analysis.

Qing LI received his master degree in Power System from CEPRI in 2006. He is now working for the renewable energy department of CEPRI. His research interests include power performance testing, power quality testing, LVRT testing, and grid adaptability testing of wind turbines and wind farms.

Chen CHEN received his master degree in Motor and electric appliances from Taiyuan University of Technology in 2009. He is now working for the renewable energy department of CEPRI. His special fields of interest include LVRT testing and grid adaptability testing of wind turbines. 